\title{
SYMMETRIZATION OF DISTRIBUTIONS AND ITS APPLICATION
}

\author{
BY \\ KUANG-HO CHEN
}

\begin{abstract}
Let $P$ be a polynomial such that $k$ of the $n-1$ principal curvatures are different from zero at each point of $N(P)=\left\{s \in R^{n}: P(s)=0\right\} ; N(P)$ is assumed to be nonempty, bounded, and $n-1$ dimensional. If Supp $\varphi \subset U^{\delta}=\left\{s \in R^{n}:|P(s)|<\delta\right\}$ with $\delta$ small and $\varphi \in C_{c}^{\infty}\left(R^{n}\right)$, let $\varphi^{\rho}$ be the integral of $\varphi$ over $N(P-q)$ if $q \in[-\delta, \delta]$ and $\varphi^{\sigma}(s)=\varphi^{\rho}(P(s))$ on $U^{\delta}$ and $=0$ outside $U^{\delta}$. Then $\varphi^{\sigma} \in C_{c}^{\infty}\left(R^{n}\right)$. We define the symmetrization $v^{\sigma}$ of a distribution $v$, with Supp $v \subset U^{\delta}$, in a natural way. Setting $u=\mathscr{F}^{-1}\{v\}$ and $u_{0}=\mathscr{F}^{-1}\left\{v^{\sigma}\right\}$, we prove that $u_{0}$ is the integral of the product of $u$ with some function $w($,$) which depends only on P$. This result is used to prove a Liouville type theorem for entire solutions of $P\left(-i D_{x}\right) u(x)=f(x)$, with $f \in C_{c}^{\infty}\left(R^{n}\right)$.
\end{abstract}

Introduction. We define the concept of symmetrization $v^{\sigma}$ (Definition 1.3) of a distribution $v$ and the concept of quadratic symmetrization $w$ (Definition 1.2) associated with the real null set $N(P)$ of a polynomial $P$. It is assumed here that $N(P)$ is nonempty, bounded and without double points. In $\S 3$, we represent the inverse Fourier transform $u_{0}$ of $v^{\sigma}$ in terms of $u$ and the quadratic symmetrization $w$ (Theorem 3.1). This involves the representation of $w$ in terms of the inverse Fourier transform of some density function defined on $N(P-q),|q|<\delta$ ( $\delta$ small) (Theorem 2.1). Estimates of the behavior of $u_{0}$ and $w$ at infinity are also derived (Theorem 4.1).

In $\S 5$ we find a criterion (Theorem 5.1) for the existence of a $C_{c}^{\infty}\left(R^{n}\right)$ solution of a partial differential equation

$$
P(-i D) u=f, \quad f \in C_{c}^{\infty}\left(R^{n}\right), \quad D_{x}=\partial / \partial x .
$$

The results in the first five sections are used in the last section to derive a Liouville type theorem (Theorem 6.1) asserting that if for each irreducible factor $P_{j}$ of a polynomial $P$ such that $N\left(P_{j}\right)$ is nonempty, bounded, and $n-1$ dimensional, at each point of $N\left(P_{j}\right)$ there are at least $k_{j}$ principal curvatures, $k_{j}>0$, of $N\left(P_{j}\right)$ different from zero, and if $u$ is a solution of the equation $\left(^{*}\right)$ such that $u(x)=o\left(|x|^{-d}\right)$ as $x$ approaches infinity with $d \geqq n-1-\min _{1 \leqq j \leqq r} k_{j} / 2$, then $u$ belongs to $C_{c}^{\infty}\left(R^{n}\right)$; moreover $u=0$ if $f=0$. A result similar to Theorem 6.1 was proved by W. Littman

Presented to the Society, October 19, 1971; received by the editors August 31, 1970 and, in revised form, March 5, 1971.

AMS 1969 subject classifications. Primary 4640, 3501; Secondary 3531, 3539, 3579.

Key words and phrases. Distribution, support of a distribution, fast-decreasing function, tempered distribution, symmetrization of a distribution (or function) with respect to a manifold, diffeomorphism, Dirac-measure on a manifold, quadratic symmetrization of a polynomial, Liouville type theorem. 
[9]. Neither result includes the other. Our Theorem 6.1 has the advantage that it allows repeated factors in the polynomial $P$, while Littman's result is not restricted by the condition that $N(P)$ be bounded. Our proof is entirely different from Littman's. The idea of our proof, in the homogeneous case, is motivated by A. Friedman [3] who discusses the decay at infinity of solutions of a polynomial of the Laplacian; in his proof another type of symmetrization is employed. A symmetrization of still a different nature appears in [13].

The results of this paper are based on the author's doctoral thesis written at Northwestern University, Evanston, Illinois. He would like to express his gratitude to his advisor, Avner Friedman, for his encouragement and helpful advice and to the referee for his kindly suggestion.

1. Symmetrization of distributions. Let $(D)$ be the space of $C_{c}^{\infty}\left(R^{n}\right)$-functions with the topology defined in L. Schwartz [11] and $\left(D^{\prime}\right)$ the space of distributions. Let $S$ be the space of fast-decreasing functions and $S^{\prime}$ denote the space of tempered distributions. $\left(D^{\prime}\right)$ and $S^{\prime}$ are the conjugate spaces of $(D)$ and $S$, respectively, [2] and [11].

Definition 1.1. A polynomial $P(s)$ is said to belong to $(\pi)$ if

(a) $N(P)=\left\{s \in R^{n}: P(s)=0\right\}$ is nonempty and bounded;

(b) $\operatorname{grad} P(s) \neq 0$ on $N(P)$.

Let $U$ be a bounded neighborhood of $N(P), U^{\delta}=\{s \in U:|P(s)|<\delta\}$, and $S_{\delta}=\left\{\varphi \in S:\right.$ Supp $\left.\varphi \subset U^{\delta}\right\}$. Let $N_{0}(P-q)=N(P-q) \cap U .\left|N_{0}(P-q)\right|$ and $d S_{q}(s)$ denote the area and the surface element, respectively, of the $n-1$ dimensional manifold $N_{0}(P-q)$ in $R^{n}$. For $\varphi \in L^{1}\left(U^{\delta}\right)$, define

$$
\begin{aligned}
\varphi^{\rho}(q) & =\frac{1}{\left|N_{0}(P-q)\right|} \int_{N_{0}(P-q)} \varphi(s) d S_{q}(s) \text { if }|q|<\delta, \\
& =0 \quad \text { if }|q| \geqq \delta
\end{aligned}
$$

and

$$
\begin{aligned}
\varphi^{\sigma}(s) & =\varphi^{\rho}(P(s)) & & \text { if } s \in U^{\delta}, \\
& =0 & & \text { if } s \notin U^{\delta} .
\end{aligned}
$$

REMARK. $d S_{q}$ is defined by $d S_{q} d q=d V$ where $d V$ is the volume element of $R^{n}$. This definition coincides with the usual intrinsic definition of the area element $d S_{q}$ [4] and [15].

Lemma 1.1. For each polynomial $P \in(\pi)$, there is a neighborhood $U$ of $N(P)$ and a number $\delta_{0}>0$ such that if $0<\delta<\delta_{0}$ and if $\varphi$ belongs to $S_{\delta}$, then $\varphi^{\circ}$ is well defined, $\varphi^{\rho} \in C_{c}^{\infty}([-\delta, \delta])$, and $\varphi^{\sigma} \in S_{\delta}$.

Proof. We must show that (i) $N_{0}(P-q)$ is a $C^{\infty}$-manifold if $|q|<\delta$ and (ii) $\varphi^{\rho}$ is $C^{\infty}$. Since $\operatorname{grad} P(s) \neq 0$ on $N(P)$, for each $a \in N(P)$, there is $j=j(a), 1 \leqq j \leqq n$, such that $D_{s,} P(s) \neq 0$. By the Inverse Mapping Theorem there is a neighborhood 
$U_{a}$ of $a$ and a ball $B_{t(a), \varepsilon(a)}$ in $R^{n}$ with center $t(a)$ and radius $\varepsilon(a)>0$ such that the mapping $t=t(s)$ given by $t_{j}=P(s)$ and $t_{i}=s_{i}$, if $i \neq j, 1 \leqq i \leqq n$, is a $C^{\infty}$-diffeomorphism from $U_{a}$ onto $B_{t(x), \varepsilon(a)}$; furthermore, on $U_{a}, D_{s_{1}} P(s) \neq 0$. Denote the inverse mapping by $s=s(t)$, or by $s_{j}=s_{j}(t)$, with $s_{i}=t_{i}$ for all $i \neq j, 1 \leqq i \leqq n$. Since $N(P)$ is compact, there is a finite number of points $a^{1}, \ldots, a^{r}$ belonging to $N(P)$ such that the corresponding neighborhoods $U_{a^{1}}, \ldots, U_{a^{r}}$ form an open covering of $N(P)$. Let $U$ be the union of these sets $U_{a}$.

Let $W$ be a neighborhood of $N(P)$ whose closure is contained in $U$. For $\delta_{0}>0$ sufficiently small, $N_{0}(P-q) \subset W$ if $|q|<\delta_{0}$. Indeed, if there is no such number $\delta_{0}$, then there are sequences $\left\{q_{i}\right\} \subset R^{1}$ and $\left\{s^{i}: s^{i} \in N_{0}\left(P-q_{i}\right) \backslash W\right\}$ such that $P\left(s^{i}\right)$ $=q_{i} \rightarrow 0$ when $i \rightarrow \infty$. Hence there is a subsequence of $\left\{s^{i}\right\}$, say $\left\{s^{i}\right\}$, convergent to some point $s^{0}$. Since $W$ is open and $s^{i} \notin W, s^{0} \notin W$. On the other hand, since $P\left(s^{0}\right)$ $=\lim P\left(s^{i}\right)=0, s^{0} \in N(P) \subset W-$ a contradiction. We have thus proved that there exists $\delta_{0}>0$ such that $N_{0}(P-q) \subset W$ if $|q|<\delta_{0}$.

Therefore $U^{\delta}$ is a neighborhood of $N(P)$ and its closure $\mathrm{Cl}\left(U^{\delta}\right)$ is contained in $\bar{W}$, hence in $U$. Moreover $N_{0}(P-q)$ is a $C^{\infty}$-manifold.

Construct the partition of unity subordinate to the covering $\left\{U_{a^{i}}\right\}$ of $\mathrm{Cl}\left(U^{\delta}\right)$ in the following way [10]:

$$
\varphi_{i} \in C_{c}^{\infty}\left(R^{n}\right), \quad \varphi_{i} \geqq 0, \quad \operatorname{Supp} \varphi_{i} \subset U_{a^{i}}, \quad \text { and } \sum_{1 \leqq i \geqq r} \varphi_{i}=1 \quad \text { on } \mathrm{Cl}\left(U^{\delta}\right) .
$$

Then for $\varphi \in S_{\delta}$,

(1) $\int_{N_{0}(P-q)} \varphi(s) d S_{q}(s)=\sum_{1 \leqq i \leqq r} \int_{N_{0}(P-q) \cap U_{a^{i}}} \varphi(s) \varphi_{i}(s) d S_{q}(s) \equiv \sum_{1 \leqq i \leqq r} \Psi_{i}(q)$.

Consider a term $\Psi_{i}$. For simplicity we assume that $j=j(a)=1$. The image of $U_{a^{i}} \cap N_{0}(P-q)$ under the $C^{\infty}$-diffeomorphism $t=t(s)$ is

$$
\left\{\left(q, t^{\prime}\right)=\left(q, t_{2}, \ldots, t_{n}\right):\left|t^{\prime}-\left(a^{i}\right)^{\prime}\right|^{2}<\varepsilon^{2}\left(a^{i}\right)-q^{2}\right\} .
$$

The inverse mapping $s=s\left(q, t^{\prime}\right)$ is given by $s_{1}=s_{1}\left(q, t^{\prime}\right), s^{\prime}=t^{\prime}$, and the Jacobian of the inverse mapping is equal to $1 / D_{s_{1}} P(s) ; D_{s_{1}} P(s) \neq 0$ on $U_{a^{i}}$. From the definition of $d S_{q}, d S_{q}=\left(1 / D_{s_{1}} P(s)\right) d t^{\prime}$. Hence

$$
\Psi_{i}(q)=\left.\int_{\left|t^{\prime}-\left(a^{i}\right)^{\prime}\right|<\left[\varepsilon^{2}\left(a^{i}\right)-q^{2}\right]^{1 / 2}} \frac{\varphi(s) \varphi_{i}(s)}{D_{s_{1}} P(s)}\right|_{s=\left(s_{1}\left(q, t^{\prime}\right), t^{\prime}\right)} d t^{\prime} .
$$

Then $\Psi_{i}(q)$ is a $C^{\infty}$-function in $q$, and hence the left-hand side of (1) is $C^{\infty}$ if $|q|$ $<\delta_{0}$. If we take $\varphi=1$ in (1), the integral is $\left|N_{0}(P-q)\right|$ which is a $C^{\infty}$-function of $q$, $|q|<\delta_{0}$. This implies that $\varphi^{\rho} \in C_{c}^{\infty}([-\delta, \delta])$ and completes the proof of the lemma.

Definition 1.2. For any function $\varphi \in S_{\delta}, 0<\delta<\delta_{0}, \varphi^{\sigma}$ is called the symmetrization of $\varphi$ with respect to the manifold $N(P)$ in the function sense.

Let $\chi_{0}$ be a function in $C_{c}^{\infty}\left(R^{1}\right)$ such that $\chi_{0} \geqq 0, \chi_{0}=1$ on $[-\delta, \delta], 0<\delta<\delta_{0}$, and Supp $\chi_{0} \subset\left(-\delta_{0}, \delta_{0}\right)$. Let $\chi(s)$ be equal to $\chi_{0}(P(s))$ on $U^{\delta_{0}}$ and 0 outside $U^{\delta_{0}}$. Then $\chi$ belongs to $S_{\delta_{0}}$. For any distribution $T$ with support in $U^{\delta}$, define the symmetrization $T^{\sigma}$ of the distribution $T$ with respect to $N(P)$ as follows:

$$
\left(T^{\sigma}, \varphi\right)=\left(T,[\chi \varphi]^{\sigma}\right), \quad \varphi \in S
$$


One easily sees that if $f$ is a continuous function with support in $U^{\delta}$, then the symmetrization of $f$ in the sense of distribution is the same as the symmetrization of $f$ in the function sense.

LEMMA 1.2. Every tempered distribution $v$ with compact support can be expressed in the form

$$
v(s)=D_{s}^{1} \mu(s) \equiv \partial^{n} \mu(s) /\left(\partial s_{1} \cdots \partial s_{n}\right) \quad(1=(1, \ldots, 1)),
$$

where $\mu$ belongs to $L^{\infty}\left(R^{n}\right)$ with support contained in

$$
G_{b+\varepsilon}=\left\{s \in R^{n}:\left|s_{j}\right|<b_{j}+\varepsilon_{j}, j=1, \ldots, n\right\}, \quad \varepsilon>0,
$$

where $b>0$ is any vector such that Supp $v \subset G_{b}$.

Proof. It is well known [2] that if $v$ in $S^{\prime}$ has compact support, then its inverse Fourier transform $u(x)$ can be extended to an entire function $u(z)$ of exponential type $\leqq b$. From [2, p. 163] or [6, p. 157], there is a completely additive complex measure $d v$ with support contained in the bounded neighborhood $G_{b+\varepsilon}$ of Supp $v$ such that

$$
u(z)=\int_{G_{b+\varepsilon}} e^{i z \cdot s} d \nu(s), \quad \varepsilon>0 .
$$

This implies that the Fourier transform of $u$ is the measure $d v$, i.e. $v=\nu$.

For any $\varphi \in C_{c}^{\infty}\left(G_{b+\varepsilon}\right)$,

Since

$$
(v, \varphi)=\int_{G_{b}+\varepsilon} \varphi(s) d \nu(s)
$$

$$
\varphi(t)=\int_{-\infty}^{t_{1}} \cdots \int_{-\infty}^{t_{n}} D_{s}^{1} \varphi(s) d s_{1} \cdots d s_{n}
$$

we have

$$
\sup _{s \in R^{n}}\{|\varphi(t)|\} \leqq\left\|D^{1} \varphi\right\|_{L^{1}\left(G_{b}+\varepsilon\right)} .
$$

Moreover, denoting by $|\nu|$ the total variation of $\nu$, we have

$$
|(v, \varphi)| \leqq \sup _{s \in G_{b+\varepsilon}}\{|\varphi(s)|\} \cdot|\nu|\left(G_{b+\varepsilon}\right) \leqq C\left\|D^{1} \varphi\right\|_{L^{1}\left(G_{b}+\varepsilon\right)} .
$$

So the map $D^{1} \varphi \rightarrow(v, \varphi)$ is a bounded linear functional on $D^{1} C_{c}^{\infty}\left(G_{b+\varepsilon}\right)$, as a subspace of $L^{1}\left(G_{b+\varepsilon}\right)$. By the Hahn-Banach Theorem it can be extended to a bounded linear functional on $L^{1}\left(G_{b+\varepsilon}\right)$. Therefore there exists a function $f \in L^{\infty}\left(G_{b+\varepsilon}\right)$ such that $\left(f, D^{\mathbf{1}} \varphi\right)=(v, \varphi)$ for all $\varphi \in C_{c}^{\infty}\left(G_{b+\varepsilon}\right)$. Hence $v=D^{\mathbf{1}} \mu$, $\mu=(-1)^{n} f$. This is the assertion of the lemma.

Definition 1.3. For any nonnegative integer $h$ we define the Dirac-measures $\left(D^{h} \delta\right)(P)$ on $N(P)$ as follows:

$$
\left(\left(D^{h} \delta\right)(P), \varphi\right)=\left(D^{h} \delta,\left[\chi^{2} \varphi\right]^{\rho}\right) \text { for any } \varphi \in S,
$$

where $\delta$ on the right-hand side is the Dirac-measure in $R^{1}$. 
THEOREM 1.1. Let the polynomial $P$ belong to the class $(\pi)$. If $v$ is a distribution with support contained in $N(P)$, then the support of $v^{\sigma}$ is in $N(P)$ and $v^{\sigma}$ is a linear combination of Dirac-measures on $N(P)$,

$$
v^{\sigma}=\sum_{0 \leqq h \leqq n} C_{h}\left(D^{h} \delta\right)(P), \quad C_{h}=\frac{(-1)^{h}}{h !}\left(v, \chi^{2} P^{h}\right) .
$$

Proof. The first assertion is obvious. We shall prove the second assertion. For any $\varphi \in S$, set

$$
\left[\chi^{2} \varphi\right]_{n}^{\rho}(q)=\left[\chi^{2} \varphi\right]^{\rho}(q)-\sum_{0 \leqq h \leqq n} \frac{q^{h}}{h !} D_{q}^{h}\left[\chi^{2} \varphi\right]^{o}(0), \quad|q|<\delta,
$$

$\left[\chi^{2} \varphi\right]_{n}^{\sigma}(s)=\left[\chi^{2} \varphi\right]_{n}^{\rho}(P(s))$ on $U^{\delta}$ and $=0$ if $s \notin U^{\delta}$. We retain the notation in the proof of Lemma 1.1. On each $U_{a^{i}}, t_{j}=P(s), t_{m}=s_{m}, m \neq j=j(i), 1 \leqq m, j \leqq n$, and we see that

$$
D_{s}^{\alpha}=\sum_{1 \leqq h \leqq|\alpha|} a_{h}(s) D_{t,}^{h}+Q\left(s, D_{t}\right)
$$

where $a_{h} \in C^{\infty}\left(U^{\delta}\right)$ and $Q\left(s, D_{t}\right)$ is the differential operator of which each term contains a factor different from $D_{t_{j}}$. Hence on $U_{a^{i}}$,

$$
D_{s}^{\alpha}\left[\chi^{2} \varphi\right]_{n}^{\sigma}(s) \equiv 0, \quad|\alpha| \leqq n .
$$

By Lemma 1.2 the order of $v$ is $\leqq n$. Since the support of $v$ is compact, by a result in [2, p. 65] $(v, \varphi)=0$ for any $\varphi \in(D)$ for which $D^{\alpha} \varphi=0$ on Supp $v$ for $|\alpha| \leqq n$. Therefore (3) implies that $\left(v,\left[\chi^{2} \varphi\right]_{n}^{\sigma}\right)=0$. Hence

$$
\begin{aligned}
\left(v^{\sigma}, \varphi\right) & =\left(v,\left[\chi^{2} \varphi\right]^{\sigma}\right)=\sum_{0 \leqq h \leqq n} \frac{1}{h !} D_{q}^{h}\left[\chi^{2} \varphi\right]^{\rho}(0)\left(v, \chi^{2} P^{h}\right) \\
& =\sum_{0 \leqq h \leqq n} \frac{1}{h !}\left(\delta(q), D_{q}^{h}\left[\chi^{2} \varphi\right]^{\rho}(q)\right)\left(v, \chi^{2} P^{h}\right) \\
& \equiv\left(\sum_{0 \leqq h \leqq n} C_{h}\left(D^{h} \delta\right)(P), \varphi\right), \quad \varphi \in S,
\end{aligned}
$$

by Definition 1.3. This yields (2).

\section{Quadratic symmetrization.}

Definition 2.1. Let $P$ be a polynomial in $(\pi)$. Define $E(s, x)=\chi(s) e^{i x \cdot s}$; then $E^{\rho}(q, x)$ and $E^{\sigma}(s, x)$ are defined for each $x \in R^{n}$. We call the inverse Fourier transform $w(, x)$ of $\chi E^{\sigma}(, x)$ the quadratic symmetrization of the polynomial $P$.

LEMMA 2.1. If $w$ is the quadratic symmetrization of the polynomial $P \in(\pi)$, then

$$
w(x, y)=\int_{-\delta}^{\delta}\left|N_{0}(P-q)\right| E^{o}(q, x) E^{o}(q, y) d q=w(y, x)
$$

for all $(x, y) \in R^{n} \times R^{n}$. 
Proof. Since $\chi E^{\sigma}(, y) \in S_{\delta_{0}}$, by Definition 2.1,

$$
\begin{aligned}
w(x, y) & =\mathscr{F}^{-1}\left\{\chi E^{\sigma}(, y)\right\}(x)=\int_{U^{\delta_{0}}} e^{i x \cdot s} \chi(s) E^{\sigma}(s, y) d s \\
& =\int_{-\delta_{0}}^{\delta_{0}}\left\{\int_{N_{0}(P-q)} e^{i x \cdot s} \chi(s) d S_{q}(s)\right\} E^{o}(q, y) d q \\
& =\int_{-\delta_{0}}^{\delta_{0}}\left|N_{0}(P-q)\right| E^{\rho}(q, x) E^{o}(q, y) d q .
\end{aligned}
$$

Definition 2.2. Let $\left(\pi_{k}\right)$ be the subclass of $(\pi)$ consisting of polynomials satisfying the condition:

(c) At each point of $N(P)$ there are at least $k$ principal curvatures of $N(P)$ distinct from zero.

Lemma 2.2. Let the polynomial $P$ belong to the class $\left(\pi_{k}\right)$. If $\delta_{0}>0$ is sufficiently small then, $P-q$ is in $\left(\pi_{k}\right)$ for each $q,|q|<\delta_{0}$.

Proof. We retain the notation in the proof of Lemma 1.1. It suffices to prove the assertion at all the points which belong to $U_{a}, a \in N(P)$. For simplicity let $j(a)=1$. Since $s=\left(s_{1}\left(q, t^{\prime}\right), t^{\prime}\right)$ is a $C^{\infty}$-diffeomorphism which maps $B_{t(a), \varepsilon(a)}$ onto $U_{a}$, where $t^{\prime}=s^{\prime}$, we can write $s\left(q, s^{\prime}\right)=\left(s_{1}\left(q, s^{\prime}\right), s^{\prime}\right)$ to express a point in $N_{0}(P-q) \cap U_{a}$ with $s_{1} \in C^{\infty}\left(B_{t(a), \varepsilon(a)}\right)$. Denote by $s^{i}\left(q, s^{\prime}\right)$ the derivative of the vector $s\left(q, s^{\prime}\right)$ with respect to $s_{i}$ and let $s^{i j}\left(q, s^{\prime}\right)=D_{s_{i}} D_{s_{j}} s\left(q, s^{\prime}\right), 2 \leqq i, j \leqq n$. Let $\kappa\left(q, s^{\prime}\right)$ be the normal of $N_{0}(P-q)$ at the point $\left(s_{1}\left(q, s^{\prime}\right), s^{\prime}\right)$. It is well known that

$$
\kappa\left(q, s^{\prime}\right)=\operatorname{grad} P\left(s_{1}\left(q, s^{\prime}\right), s^{\prime}\right) /\left|\operatorname{grad} P\left(s_{1}\left(q, s^{\prime}\right), s^{\prime}\right)\right| .
$$

Let $L_{i j}\left(q, s^{\prime}\right)$ be the scalar product of the normal with $s^{i j}\left(q, s^{\prime}\right)$. Then

$$
L_{i j} \in C^{\infty}\left(B_{t(a), \varepsilon(a)}\right)
$$

By definition, the $n-1$ principal curvatures $\omega_{i}\left(q, s^{\prime}\right)$ of $N_{0}(P-q)$ at the point $\left(s_{1}\left(q, s^{\prime}\right), s^{\prime}\right)$ are the $n-1$ eigenvalues of the matrix $\left(L_{i j}\left(q, s^{\prime}\right)\right)$. But the determinant of the matrix $\left(\lambda \delta_{i j}-L_{i j}\left(q, s^{\prime}\right)\right)$ is a polynomial in $\lambda$ of degree $n-1$; i.e.

$$
\operatorname{det}\left(\lambda \delta_{i j}-L_{i j}\left(q, s^{\prime}\right)\right)=\lambda^{n-1}+\sum_{1 \leqq j<n} H_{j}\left(q, s^{\prime}\right) \lambda^{n-j-1},
$$

where $H_{j} \in C^{\infty}\left(B_{t(a), \varepsilon(a)}\right)$. Moreover, for $1 \leqq i<n$,

$$
H_{i}\left(q, s^{\prime}\right)=(-1)^{i} \sum_{1 \leqq j_{1}<\cdots<j_{i}<n} \omega_{j_{1}}\left(q, s^{\prime}\right) \cdots \omega_{j_{i}}\left(q, s^{\prime}\right) .
$$

By condition (c) we can assume that the number of nonzero principal curvatures of $N(P)$ at $a$ is $h$ with $k \leqq h<n$. Hence $H_{i}\left(q, s^{\prime}\right)=0$ at $a$ for all $h<i<n$ and $H_{h}\left(q, s^{\prime}\right)$ $\neq 0$ at $s=a$. Since $H_{h} \in C^{\infty}\left(B_{t(a), \varepsilon(a)}\right)$, there is a neighborhood, say $U_{a}$, of $a$ on which $H_{h}\left(q, s^{\prime}\right) \neq 0$. Using the Heine-Borel theorem, we obtain the assertion of the lemma. 
From Definition 2.1 we see that $E^{o}(q$,$) is the inverse Fourier transform of$ $\delta(P-q)$ if $|q|<\delta_{0}$. Hence by the result of [8] (with correction in [9]) and by the assertion of Lemma 2.2, we obtain the next lemma.

Lemma 2.3. Let the polynomial $P$ belong to the class $\left(\pi_{k}\right)$. If $k=n-1$, then for each $|q|<\delta_{0}$ and for each unit vector $\omega$, there is only a finite number of points $p_{j}(q, \omega)$ on $N_{0}(P-q)$ at which the normal of $N_{0}(P-q)$ is $\omega ;$ further, for $x \in R^{n},|x| \omega \rightarrow \infty$,

$$
\begin{aligned}
E^{\rho}(q, x)=\left\{|x|^{-(n-1) / 2}\left(\frac{\pi}{2}\right)^{(n-1) / 2} \sum_{j} \frac{(1+i)^{d_{+}\left(p_{j}(q, \omega)\right)}(1-i)^{d_{-}\left(p_{j}(q, \omega)\right)}}{\left|K_{n-1}\left(p_{j}(q, \omega)\right)\right|^{1 / 2}}\right. \\
\left.\quad \times \exp \left(i p_{j}(q, \omega) \cdot x\right)+O\left(|x|^{-n / 2}\right)\right\} \frac{\chi_{0}(q)}{\left|N_{0}(P-q)\right|} .
\end{aligned}
$$

If $k<n-1$, then for each $|q|<\delta_{0}$ there is a nonempty open set $\Omega(q)$ of unit vectors such that, for each $\omega \in \Omega(q)$, there is only a finite numbers of points $p_{j}(q, \omega)$ on $N_{0}(P-q)$ with neighborhoods $U_{j}=\left\{p(\xi) \in N_{0}(P-q): \xi \in S_{j}(q, \omega)\right\}$ at which the normal $\kappa(p(\xi))$ is $\omega$ for all $j$. Here $S_{j}(q, \omega)$ are open subsets of $R^{n-1-k}$. Moreover, as $|x| \omega \rightarrow \infty$,

$$
\begin{aligned}
E^{o}(q, \omega)=\left(\frac{\pi}{2}|x|^{-1}\right)^{k / 2}\left\{\sum_{j}\left|S_{j}(q, \omega)\right|\right. & \frac{(1+i)^{d_{+}\left(p_{j}(q, \omega)\right)}(1-i)^{d_{-}\left(p_{j}(q, \omega)\right)}}{\left|K_{k}\left(p_{j}(q, \omega)\right)\right|^{1 / 2}} \\
& \left.\times \exp \left(i p_{j}(q, \omega) \cdot x\right)+O\left(|x|^{-(k+1) / 2}\right)\right\} \frac{\chi_{0}(q)}{\left|N_{0}(P-q)\right|} .
\end{aligned}
$$

Here $\left|S_{j}(q, \omega)\right|$ is the volume of $S_{j}(q, \omega)$ in $R^{n-1-k}, K_{k}\left(p_{j}(q, \omega)\right)$ is the product of the $k$ nonzero principal curvatures of $N_{0}(P-q)$ at $p_{j}(q, \omega), d_{+}\left(p_{j}(q, \omega)\right)$ and $d_{-}\left(p_{j}(q, \omega)\right)$ are the numbers of the positive and negative principal curvatures of $N_{0}(P-q)$ at $p_{j}(q, \omega)$, respectively. (Notice that $K_{k}(t) \equiv 1$ if $k=0$.)

THEOREM 2.1. Let the polynomial P belong to the class $\left(\pi_{k}\right)$. Then for each pair of unit vectors $\omega$ and $\bar{\omega}$ and any integer $p \geqq 0$,

$$
w(|x| \omega,|y| \bar{\omega})=O\left(|x|^{-p-k / 2}|y|^{p-k / 2}[|x|+|y|]^{-1}\right),
$$

as $(|x| \omega,|y| \bar{\omega}) \rightarrow \infty$ in the direction $(\omega, \bar{\omega})$. Here we assume that $k$ is the small integer such that the class $\left(\pi_{k}\right)$ contains $P$.

In the definition of $w$ we took $0<\delta<\delta_{0}$. In the proof of Theorem 2.1 we need $\delta_{0}$ to be a sufficiently small number (independent of $p_{j}, \omega$ and $\bar{\omega}$ ). We shall need

LEMMA 2.4. Let the conditions of Lemma 2.3 with $k=n-1$ hold and suppose that $D_{s_{1}} P(s) \neq 0$ at $p_{j}(0, \omega)$. Then

$$
p_{j}(q, \omega)=\left(s_{1}\left(q, s^{\prime}\left(q, \omega^{\prime}\right)\right), s^{\prime}\left(q, \omega^{\prime}\right)\right), \quad q \in\left[-\delta_{0}, \delta_{0}\right],
$$

where $s^{\prime}\left(q, \omega^{\prime}\right)=\left(f_{2}\left(q, \omega^{\prime}\right), \ldots, f_{n}\left(q, \omega^{\prime}\right)\right)$ and $f_{j}(,) \in C^{\infty}\left(\left[-\delta_{0}, \delta_{0}\right] \times U\right)$ for some neighborhood $U \subset R^{n-1}$ of $\omega^{\prime}$ and some number $\delta_{0}>0$. 
Proof. With the notation in the proof of Lemma 2.2, we first prove that the $(n-1) \times n$ matrix

$$
\Lambda(s)=\left(\begin{array}{c}
D_{s_{2}} \kappa_{1}(s), \ldots, D_{s_{2}} \kappa_{n}(s) \\
\ldots \ldots \ldots, \ldots, \ldots, \ldots, \ldots, D_{s_{n}} \kappa_{n}(s)
\end{array}\right)
$$

has rank $n-1$ with $s=\left(s_{1}\left(q, s^{\prime}\right), s^{\prime}\right)$ in $U^{\delta}$, for some small $\delta>0$, where $\kappa(s)$ $=\left(\kappa_{1}(s), \ldots, \kappa_{n}(s)\right)$. Indeed, since the $n-1$ principal cruvatures of $N_{0}(P-q)$ at the point $s$ are the $n-1$ eigenvalues of the matrix $\left(L_{i j}(s)\right)$, by condition (c) with $k=n-1$, the matrix $\left(L_{i j}(s)\right)$ is nonsingular; i.e. its rank is $n-1$ for all $s \in U^{\delta}$ with the number $\delta>0$ satisfying the assertion of Lemma 2.2. But on the other hand $\kappa(s) \cdot D_{s_{i}} s(s)=0, i=2, \ldots, n$. Hence for $1<i, j \leqq n$,

$$
L_{i j}(s)=-D_{s^{\prime}} k(s) \cdot D_{s_{i}} s(s)
$$

Let $\Gamma(s)$ be the $n \times(n-1)$ matrix with $-D_{s_{i}} s(s)$ as its $(i+1)$ th column, $1<i \leqq n$. Then $\left(L_{i j}(s)\right)=\Lambda(s) \cdot \Gamma(s)$, and therefore $\Lambda(s)$ is of rank $n-1$ for each $s \in U^{\delta}$.

At $p_{j}(0, \omega)=\left(s_{1}\left(0, s_{0}^{\prime}\right), s_{0}^{\prime}\right)=s_{0}$, we can assume the last $n-1$ columns of $\Lambda\left(s_{0}\right)$, denoted by $\Lambda^{*}\left(s_{0}\right)$, are linearly independent; i.e. the $(n-1) \times(n-1)$ matrix $\Lambda^{*}\left(s_{0}\right)$ is nonsingular.

Let

$$
\begin{aligned}
& F_{i}\left(s^{\prime}, q, \kappa^{\prime}\right)=\kappa_{i+1}\left(s_{1}\left(q, s^{\prime}\right), s^{\prime}\right), \quad i=1, \ldots, n-1, \\
& F_{n}\left(s^{\prime}, q, \kappa^{\prime}\right)=q
\end{aligned}
$$

and denote by $J_{F}\left(s, q, \kappa^{\prime}\right)$ the Jacobian of

$$
F\left(s^{\prime}, q, \kappa^{\prime}\right)=\left(F_{1}\left(s^{\prime}, q, \kappa^{\prime}\right), \ldots, F_{n}\left(s^{\prime}, q, \kappa^{\prime}\right)\right)
$$

with respect to the variables $\left(s^{\prime}, q\right)$. Then since $J_{F}\left(s^{\prime}, q, \kappa^{\prime}\right)=\operatorname{det} \Lambda^{*}(s)$ at $s=s_{0}$, by the Implicit Function Theorem there is a number $\delta_{0}>0$ and a neighborhood $U \subset R^{n-1}$ of $\omega^{\prime}$ such that

$$
s^{\prime}\left(q, \kappa^{\prime}\right)=\left(f_{2}\left(q, \kappa^{\prime}\right), \ldots, f_{n}\left(q, \kappa^{\prime}\right)\right)
$$

with $f_{j}(,) \in C^{\infty}\left(\left[-\delta_{0}, \delta_{0}\right] \times U\right)$. This completes the proof of the lemma.

Proof of Theorem 2.1. (For the case $k=n-1$.) By condition (b), we can assume $D_{s_{1}} P(s) \neq 0$ at $p_{j}(0, \omega)$. Then Lemma 2.4 yields

$$
q=P\left(p_{j}(q, \omega)\right)=P\left(f_{1}\left(q, \omega^{\prime}\right), \ldots, f_{n}\left(q, \omega^{\prime}\right)\right), \quad q \in\left[-\delta_{0}, \delta_{0}\right],
$$

with $f_{1}\left(, \omega^{\prime}\right)=s_{1}\left(, s^{\prime}\left(, \omega^{\prime}\right)\right) \in C^{\infty}\left(\left[-\delta_{0}, \delta_{0}\right]\right)$. This implies

$$
1=D_{q} P\left(p_{j}(q, \omega)\right)=\operatorname{grad} P\left(p_{j}(q, \omega)\right) \cdot D_{q} p_{j}(q, \omega) .
$$

Hence this and (4) imply

$$
D_{q}\left[p_{j}(q, \omega) \cdot \omega\right]=D_{q} p_{j}(q, \omega) \cdot \omega=1 /\left|\operatorname{grad} P\left(p_{j}(q, \omega)\right)\right| .
$$


By Lemma 2.3 and the definition of the quadratic symmetrization of the polynomial $P$,

$$
\begin{aligned}
w(|x| \omega,|y| \bar{\omega})= & (|x||y|)^{-(n-1) / 2} \\
& \times \sum_{h, j} \int_{-\delta}^{\delta} B_{n-1, h, j}(q, \omega, \bar{\omega}) \exp \left\{i p_{j}(q, \omega) \cdot x+i p_{h}(q, \bar{\omega}) \cdot y\right\} d q \\
& +\int_{-\delta}^{\delta} O\left([|x||y|]^{-n / 2}\right) d q,
\end{aligned}
$$

when $(|x| \omega,|y| \bar{\omega}) \rightarrow+\infty$ with $\omega$ and $\bar{\omega}$ fixed, where the summation is independent of $q$ and

$$
\begin{aligned}
B_{n-1, h, j}(q, \omega, \bar{\omega})=\left(\frac{\pi}{2}\right)^{n-1} \frac{\chi_{0}^{2}(q)}{\left|N_{0}(P-q)\right|^{2}} \\
\\
\quad \times \frac{(1+i)^{d_{+}\left(p_{j}(q, \omega)\right)+d_{+}\left(p_{h}(q, \bar{\omega})\right)}(1-i)^{d_{-}\left(p_{j}(q, \omega)\right)+d_{-}\left(p_{h}(q, \bar{\omega})\right)}}{\left|K_{n-1}\left(p_{j}(q, \omega)\right) K_{n-1}\left(p_{h}(q, \bar{\omega})\right)\right|^{1 / 2}} .
\end{aligned}
$$

Since the principal curvatures are continuous in $s=s(q, \omega)=\left(f_{1}(q, \omega), \ldots, f_{n}(q, \omega)\right)$ which is continuous in $q$, we can choose $\delta_{0}$ so small that $d_{+}\left(p_{j}(q, \omega)\right)$ and $d_{-}\left(p_{j}(q, \omega)\right)$ are independent of $q$ if $|q|<\delta_{0}$. Hence for each pair of unit vectors $\omega$ and $\bar{\omega}$,

$$
\begin{aligned}
& B_{n-1, h, j}(, \omega, \bar{\omega}) \in C_{c}^{\infty}\left(\left[-\delta_{0}, \delta_{0}\right]\right) \text { and } \\
& B_{n-1, h, j}(q, \omega, \bar{\omega})=0 \text { if }|q| \geqq \delta_{0} .
\end{aligned}
$$

Let the integrals on the right-hand side of (6) be denoted by $I_{h, j}(|x| \omega,|y| \bar{\omega})$. By (5)

$$
\begin{aligned}
D_{q} \exp \left\{i p_{j}(q, \omega) \cdot|x| \omega\right\} & =i|x| \exp \left(i p_{j}(q, \omega) \cdot x\right) D_{q}\left[p_{j}(q, \omega) \cdot \omega\right] \\
& =\left(i|x| /\left|\operatorname{grad} P\left(p_{j}(q, \omega)\right)\right|\right) \exp \left(i p_{j}(q, \omega) \cdot|x| \omega\right) .
\end{aligned}
$$

Integrating by parts and using (7) and (8), we have

$$
\begin{aligned}
& I_{h, j}(|x| \omega,|y| \bar{\omega})= \int_{-\delta_{0}}^{\delta_{0}} B_{n-1, h, j}(q, \omega, \bar{\omega}) \\
& \times \exp \left(i p_{h}(q, \bar{\omega}) \cdot y\right) \\
&= {\left[\frac{\left|\operatorname{grad} P\left(p_{j}(q, \omega)\right)\right|}{i|x|} D_{q} \exp \left(i p_{j}(q, \omega) \cdot x\right)\right] d q } \\
&|x| \int_{-\delta_{0}}^{\delta_{0}} B_{n-1, h, j}(q, \omega, \bar{\omega}) \frac{\left|\operatorname{grad} P\left(p_{j}(q, \omega)\right)\right|}{\left|\operatorname{grad} P\left(p_{h}(q, \bar{\omega})\right)\right|} \\
& \times \exp \left\{i p_{j}(q, \omega) \cdot x+i p_{h}(q, \bar{\omega}) \cdot y\right\} d q+O\left(|x|^{-1}\right),
\end{aligned}
$$

when $(|x| \omega,|y| \bar{\omega}) \rightarrow \infty$. Repeating the argument $p$ times, we obtain

$$
\begin{aligned}
I_{h, j}(|x| \omega,|y| \bar{\omega})= & \left(\frac{|y|}{|x|}\right)^{p} \int_{-\delta_{0}}^{\delta_{0}} B_{n-1, h, j}(q, \omega, \bar{\omega})\left[\frac{\left|\operatorname{grad} P\left(p_{j}(q, \omega)\right)\right|}{\left|\operatorname{grad} P\left(p_{h}(q, \bar{\omega})\right)\right|}\right]^{p} \\
& \quad \times \exp \left\{i p_{j}(q, \omega) \cdot x+i p_{h}(q, \bar{\omega}) \cdot y\right\} d q+O\left(|y|^{p-1}|x|^{-p}\right) \\
\equiv & \left(\frac{|y|}{|x|}\right)^{p} \int_{-\delta_{0}}^{\delta_{0}} B_{n-1, h, j}^{(p)}(q, \omega, \bar{\omega}) \\
& \quad \times \exp \left\{i p_{j}(q, \omega) \cdot x+i p_{h}(q, \bar{\omega}) \cdot y\right\} d q+O\left(|y|^{p-1}|x|^{-p}\right)
\end{aligned}
$$


when $(|x| \omega,|y| \bar{\omega}) \rightarrow \infty$, where $B_{n-1, h, j}^{(p)}$ satisfies (7). By (8),

$$
\begin{aligned}
D_{q} \exp \left\{i p_{j}(q, \omega) \cdot x+i p_{h}(q, \bar{\omega}) \cdot y\right\}=\left[\frac{i|x|}{\left|\operatorname{grad} P\left(p_{j}(q, \omega)\right)\right|}+\frac{i|y|}{\left|\operatorname{grad} P\left(p_{h}(q, \bar{\omega})\right)\right|}\right] \\
\times \exp \left\{i p_{j}(q, \omega) \cdot x+i p_{h}(q, \bar{\omega}) \cdot y\right\} .
\end{aligned}
$$

With this replacing (8) in the integration by parts, we get

$$
\begin{aligned}
I_{h, j}(|x| \omega,|y| \bar{\omega})=\left(\frac{|y|}{|x|}\right)^{p} \int_{-\delta_{0}}^{\delta_{0}} B_{n-1, h, j}^{(p)}(q, \omega, \bar{\omega}) & \\
& \times \frac{D_{q} \exp \left\{p_{j}(q, \omega) \cdot x i+i p_{h}(q, \bar{\omega}) \cdot y\right\}}{i|x| /\left|\operatorname{grad} P\left(p_{j}(q, \omega)\right)\right|+i|y| /\left|\operatorname{grad} P\left(p_{h}(q, \bar{\omega})\right)\right|} \\
& +O\left(|y|^{p-1}|x|^{-p}\right)
\end{aligned}
$$

when $(|x| \omega,|y| \bar{\omega}) \rightarrow \infty$. From the proofs in [8] (with the correction in [9]), we see that the above technique can be employed for the last term in (6). Therefore when $(x, y) \rightarrow \infty$,

$$
w(x, y)=O\left(|x|^{-(n-1) / 2-p}|y|^{p-(n-1) / 2}[|x|+|y|]^{-1}\right) .
$$

This is the assertion of Theorem 2.1 for the case $k=n-1$.

From the idea introduced in [8] and by the technique used above, we can obtain the assertion for the case $k<n-1$.

\section{A representation theorem.}

TheOREM 3.1. Let the polynomial $P$ belong to $\left(\pi_{k}\right), k>0$. Let $u$ be a distribution such that the support of its Fourier transform $v$ is contained in $U^{\delta}, 0<\delta<\delta_{0}$ with $\delta_{0}$ sufficiently small. Then the inverse Fourier transform $u_{0}$ of the symmetrization $v^{\sigma}$ of $v$ with respect to $N(P)$ can be expressed in the form

$$
u_{0}(x)=\int_{R^{n}} u(y) w(x, y) d y,
$$

where $w$ is the quadratic symmetrization of $P$.

Proof. Since $w(x)=,\mathscr{F}^{-1}\left\{\chi E^{\sigma}(, x)\right\}$, with $\chi E^{\sigma}(, x) \in S, w(x$,$) belongs to S$ for each $x \in R^{n}$. Moreover, it is a $C^{\infty}$-function in $(x, y) \in R^{n} \times R^{n}$. Let $\psi$ be any element of $S, \varphi=\mathscr{F}^{-1}\{\psi\}$, and $\psi_{0}=\mathscr{F}^{-1}\left\{\left[\chi^{2} \varphi\right]^{\sigma}\right\}$. Let $\chi_{1} \in C_{c}^{\infty}\left(R^{n}\right), \chi_{1} \geqq 0$, equal to 1 on the support of $\chi$ and with support in $U^{\delta}, 0<\delta<\delta_{0}$, with $\delta_{0}$ satisfying the assertions of Theorem 2.1. Then $\chi \chi_{1}=\chi$. This and the note made after the proof of Lemma 1.1 yield

$$
\begin{aligned}
\psi_{0}(x) & =\mathscr{F}^{-1}\left\{\left[\chi^{2} \varphi\right]^{\sigma}\right\}(x)=\left(\left[\chi^{2} \varphi\right]^{\sigma}, \chi_{1} \exp \{i x \cdot\}\right) \\
& =\left(\chi^{2} \varphi,\left[\chi_{1} \exp \{i x \cdot\}\right]^{\sigma}\right)=\left(\varphi, \chi[\chi \exp \{i x \cdot\}]^{\sigma}\right) \\
& =\left(\varphi, \chi E^{\sigma}(x,)\right)=\left(\psi, \mathscr{F}^{-1}\left\{\chi E^{\sigma}(x,)\right\}\right) \\
& =\int_{R^{n}} \psi(y) w(x, y) d y .
\end{aligned}
$$


Since the support of $v$ is compact, $u(x)$ is analytic and $|u(x)| \leqq C(1+|x|)^{m}, m \geqq 0$ and $C>0$. Hence, by this and Theorem 2.1, the integral

$$
\int_{R^{n} \times R^{n}} u(x) w(x, y) \psi(y) d(x, y)
$$

is absolutely convergent for all $\psi \in S$. Thus, (9) and Fubini's Theorem yield

$$
\begin{aligned}
\left(u_{0}, \psi\right) & =\left(\mathscr{F}^{-1}\left\{v^{\sigma}\right\}, \psi\right)=\left(v^{\sigma}, \chi^{2} \varphi\right)=\left(v,\left[\chi^{2} \varphi\right]^{\sigma}\right) \\
& =\left(u, \psi_{0}\right)=\left(\int_{R^{n}} u(y) w(x, y) d y, \psi\right), \quad \psi \in S .
\end{aligned}
$$

Hence $u_{0}(x)=\int_{R^{n}} u(y) w(x, y) d y$.

4. Decay at infinity of the inverse Fourier transform of the symmetrized distribution. Using Theorems 1.1 and 3.1, we can determine the decay at infinity of $u_{0}$, which depends on the class to which the polynomial $P$ belongs.

THEOREM 4.1. Let the support of the Fourier transform $v$ of a distribution $u$ be contained in $N(P), P \in\left(\pi_{k}\right)$ with smallest $k>0$, and let $u_{0}$ be the inverse Fourier transform of the symmetrization $v^{\sigma}$ of $v$ with respect to $N(P)$. Then, as $|x| \rightarrow \infty$, $u_{0}(x)=O\left(|x|^{-d}\right), u_{0}(x) \neq o\left(|x|^{-d}\right)$, for some $d \leqq k / 2$, provided $u_{0} \neq 0$; on the other hand, $u_{0}(x)=o\left(|x|^{n-1-k-b}\right)$ if $u(x)=o\left(|x|^{-b}\right), b \geqq 0$.

Proof. Definition 1.3 yields

$$
\begin{aligned}
\mathscr{F}^{-1}\left\{\left(D^{h} \delta\right)(P)\right\}(x) & =\left(\left(D^{h} \delta\right)(P(s)), e^{i x \cdot s}\right) \\
& =\left(\left(D_{q}\right)^{h} \delta(q),\left[\chi^{2} \exp \{i x \cdot\}\right]^{\rho}(q)\right) \\
& =\left(\left(D_{q}\right)^{h} \delta(q), E^{\rho}(q, x)\right)=\left(\delta(q),\left(-D_{q}\right)^{h} E^{\rho}(q, x)\right) .
\end{aligned}
$$

This and Theorem 1.1 imply

$$
u_{0}(x)=\mathscr{F}^{-1}\left\{v^{\sigma}\right\}(x)=\sum_{0 \leqq h \leqq n} C_{h}\left(\delta(q),\left(-D_{q}\right)^{h} E^{\rho}(q, x)\right) .
$$

First assume $k=n-1$. Lemma 2.3 implies that, when $|x| \omega \rightarrow+\infty$,

$$
\begin{aligned}
E^{\rho}(q,|x| \omega)= & |x|^{-(n-1) / 2} \sum_{j} A_{n-1}\left(p_{j}(q, \omega)\right) \cdot \exp \left(i p_{j}(q, \omega) \cdot x\right) \frac{\chi_{0}(q)}{\left|N_{0}(P-q)\right|} \\
& +O\left(|x|^{-n / 2}\right)
\end{aligned}
$$

where

$$
A_{n-1}(t)=\left(\frac{\pi}{2}\right)^{n-1} \frac{(1+i)^{d_{+}{ }^{(t)}}(1-i)^{d_{-}(t)}}{\left|K_{n-1}(t)\right|^{1 / 2}}
$$

is a $C^{\infty}$-function in $t \in R^{1}$. By Lemma $2.4 p_{j}(q, \omega)$ is a $C^{\infty}$-function in $q \in[-\delta, \delta]$, $0<\delta<\delta_{0}$, with $\delta_{0}$ satisfying the assertion of Theorem 2.1. By (5) and (11), when $|x| \omega \rightarrow \infty$,

$$
D_{q}^{h} E^{o}(q,|x| \omega)=|x|^{h-(n-1) / 2} f_{h}(q, \omega,|x|)+O\left(|x|^{h-1-(n-1) / 2}\right),
$$


for any $0 \leqq h \leqq n$, where

$$
f_{h}(q, \omega,|x|)=\sum_{j} \frac{A_{n-1}\left(p_{j}(q, \omega)\right) \cdot i^{h}}{\left|\operatorname{grad} P\left(p_{j}(q, \omega)\right)\right|^{h}} \exp \left(i p_{j}(q, \omega) \cdot|x| \omega\right) \frac{\chi_{0}(q)}{\left|N_{0}(P-q)\right|} .
$$

Hence when $|x| \omega \rightarrow \infty$,

$$
\begin{aligned}
\left(\delta(q), D_{q}^{h} E^{\rho}(q,|x| \omega)\right) & =D_{q}^{h} E^{\rho}(0,|x| \omega) \\
& =|x|^{h-(n-1) / 2} f_{h}(0, \omega,|x|)+O\left(|x|^{h-1-(n-1) / 2}\right) .
\end{aligned}
$$

For each fixed $h$ and $\omega$, we see that $f_{h}(0, \omega,|x|)$ is a uniformly almost periodic function [1] in $|x|$. If there is more than one index, we choose a suitable point in $R^{n}$ as origin to make the projection $|x| p_{j}(0, \omega) \cdot \omega$ of $|x| p_{j}(0, \omega)$ in the direction $\omega$ distinct from each other. Since

$$
\frac{A_{n-1}\left(p_{j}(0, \omega)\right) \cdot i^{h}}{\left|\operatorname{grad} P\left(p_{j}(0, \omega)\right)\right|^{h}} \cdot \frac{\chi_{0}(0)}{|N(P)|} \neq 0
$$

$f_{h}(0, \omega,|x|) \not \equiv 0$ for some fixed unit vector $\omega$ for each $h$. Hence for any $0 \leqq h \leqq n$, when $x \rightarrow+\infty$,

$$
D_{q}^{h} E^{\rho}(0, x)=O\left(|x|^{n-(n-1) / 2}\right), \quad D_{q}^{h} E^{\rho}(0, x) \neq o\left(|x|^{h-(n-1) / 2}\right) .
$$

Similar arguments using the idea introduced in [8] can be applied to the case $k<n-1$. Therefore this and (10) yield the first assertion.

For the last assertion, let $\varepsilon>0$ be given, by the condition $u(x)=o\left(|x|^{-b}\right)$ as $x \rightarrow \infty$, there exists a number $M_{\varepsilon}>0$ such that $|x|^{b}|u(x)|<\varepsilon$ if $|x|>M_{\varepsilon}$. We write the integral in Theorem 1.3 as the sum of three integrals whose integration ranges are $|y| \leqq M_{\varepsilon}, M_{\varepsilon} \leqq|y| \leqq|x|$, and $|x| \leqq|y|$. Then this and Theorem 2.1 show that $u_{0}(x)$ is bounded by

$$
C_{1} M_{\varepsilon}^{p+n-k / 2}|x|^{-k / 2-p-1}+C_{2}|x|^{n-1-b-k}+C_{3}|x|^{n-b-k-1},
$$

when $|x|>M_{\varepsilon}$ and $p$ is an integer $>\max \{0, b+k / 2+1-n, n-b-k / 2\}$. Hence we obtain the assertion.

5. A criterion for existence of solutions of nonhomogeneous equations. The result in this section is of interest in itself. At the same time, we provide an application in the next section.

Definition 5.1. Let $(\Pi)$ denote the class of the polynomials

$$
P(s)=P_{0}(s) \prod_{1 \leqq j \leqq r}\left[P_{j}(s)\right]^{r_{j}}, \quad s \in R^{n},
$$

satisfying conditions:

(i) $N\left(P_{0}\right)=\varnothing$;

(ii) $P_{j} \in(\pi)$ and is irreducible for each $1 \leqq j \leqq r$;

(iii) no two of manifolds. $N\left(P_{j}\right), 1 \leqq j \leqq r$, are equal. 
By the definition, if $P \in(\Pi)$, then for each $\alpha \in N(P)$, there is an integer $k_{a}$, $1 \leqq k_{a} \leqq r$, such that $a$ is in $N_{i_{1} \ldots i_{k_{a}}}=N\left(P_{i_{1}}\right) \cap \cdots \cap N\left(P_{i_{k_{a}}}\right), 1 \leqq i_{1}<\cdots<i_{k_{a}} \leqq r$, and $a \notin N\left(P_{i}\right)$ if $i \neq i_{1}, \ldots, i_{k_{a}}$; for simplicity, assume $i_{j}=j$. Condition (b) in the definition of $(\pi)$ shows that there is an integer $\nu=\nu(a, j), 1 \leqq \nu \leqq n$, such that $D_{s_{v}} P_{j}(s) \neq 0$ at $s=a$. By the Inverse Mapping Theorem there is a $C^{\infty}$-diffeomorphism $t=t(s): t_{v}$ $=P_{j}(s), t_{m}=s_{m}, m \neq v, 1 \leqq m \leqq n$, which maps a neighborhood $U_{a, j}$ of $a$ onto a neighborhood $V_{t(a), j}$ of $t(a)$, and whose inverse mapping $s=s(t)$ is given by $s_{v}=s_{v}(t) \in C^{\infty}\left(V_{t(a), j}\right), s_{m}=t_{m}$. For any $\varphi \in C^{\infty}\left(R^{n}\right)$, we can define, on $U_{a, j}$,

$$
\left.D_{j}^{h} \varphi(s) \equiv D_{P_{j}}^{h} \varphi(s) \equiv D_{t_{v}}^{h} \varphi\left(t_{1}, \ldots, t_{v-1}, s_{v}(t), t_{v+1}, \ldots, t_{n}\right)\right|_{t=t(s)} .
$$

Then on $U_{a}=\bigcap_{1 \leqq j \leqq k_{a}} U_{a, j}, D_{i}^{k_{i}} \ldots D_{1}^{k_{1}} \varphi(s)$ is meaningful.

Definition 5.1. With $P \in(\Pi)$ denote by $\left(C_{P}\right)$ the class of functions $f \in(D)$ which satisfy the condition

(A) For $1 \leqq i_{1}<\cdots<i^{d} \leqq r$, at each point of $N_{i_{1} \ldots i_{d}}, D_{i_{d}}^{h_{d}} \cdots D_{i_{1}}^{h_{1}}[\mathscr{F}\{f\}(s)]=0$, $0 \leqq h^{d}<r^{d}, 0 \leqq h_{j} \leqq r, 1 \leqq j<d$.

TheOREM 5.1. With $P \in(\Pi)$, we have $P(-i D)(D)=\left(C_{P}\right)$.

Proof. It suffices to show that (A) is the necessary and sufficient condition about the existence of a solution $u \in(D)$ of

$$
P(-i D) u=f, \quad f \in(D),
$$

with $f \neq 0$. We assume first that $u \in(D)$ is a solution of (12) with $f \neq 0$. Then $v=\mathscr{F}\{u\} \in S$ and (12) yields

$$
g(s) \equiv \mathscr{F}\{f\}(s)=P(s) v(s)=P_{0}(s) \prod_{1 \leqq j \leqq r}\left[P_{j}(s)\right]^{r} v(s) .
$$

Hence condition (A) follows from the definition of $D_{i} \varphi$ and direct calculation.

Next we assume that the condition (A) holds. We require the following [12]

Lemma 5.1. Let $Q(s)$ be an irreducible polynomial with complex coefficients and let $\mathfrak{N}(Q)$ be the set of zeros of $Q(z)$ in n-dimensional complex space $C^{n}$. Let $\varphi$ be an entire function in $C^{n}$. Assume that the function $\varphi / Q$ is defined in $C^{n}$ and $\varphi / Q$ can be extended as a holomorphic function to an open set $\Gamma$ intersecting $\mathfrak{R}(Q)$. Then $\varphi / Q$ can be extended to $C^{n}$ as an entire function.

Pick a point $a \in N\left(P_{1}\right)$. The set of complex zeros of $P_{1}$ restricted to a complex neighborhood $U$ of $a$ is an analytic set of complex dimension $n-1$. Since $f(x)$ has compact support, $g=\mathscr{F}\{f\}$ can be extended to an entire function $g(s+i t)$ of exponential type. The set $\Lambda$ of common zeros of $P_{1}(s+i t)$ and $g(s+i t)$ in $U$, with small $U$, either coincides with the set of zeros of $P_{1}(s+i t)$ in $U$ or has complex dimension $\leqq n-2$. In the latter case the restriction of $\Lambda$ to $R^{n}$ must have real dimension $\leqq n-2$. But condition (b) of Definition 1.1 and (A) show that the restriction is $n-1$ dimensional. We have a contradiction. Hence whenever $P_{1}(s+i t)$ vanishes in $U, g(s+i t)$ must also vanish. From this and condition (b) at the point $a$, 
it follows that $g(s+i t) / P_{1}(s+i t)$ can be extended as a holomorphic function to a complex neighborhood of $a$. Using Lemma 5.1, we see that $g_{1}(s)=g(s) / P_{1}(s)$ can be extended to all of $C^{n}$ as an entire function. It is well known [12] that this function must be of exponential type and of the same type of $g$. This implies that $f_{1}=\mathscr{F}^{-1}\left\{g_{1}\right\}$ must have compact support. Now, $P_{1}(s) g_{1}(s)=P_{1}(s) g(s) / P_{1}(s)=g(s)$, so that we have $P_{1}(-i D) f_{1}=f$. This and the definitions of $f_{1}$ and $g_{1}$ show that $f_{1}$ satisfies condition (A) with $r_{1}$ replaced by $r_{1}-1$.

Next we consider (12) with $f$ replaced by $f_{1}$ and $P$ replaced by $P / P_{1}$. Repeating the above arguments $r_{1}+\cdots+r_{r}-1$ times, we obtain a function $u_{r} \in(D)$ which satisfies (12) with $f=u_{r}$; i.e.

$$
P_{0}(-i D) u=u_{r}, \quad u_{r} \in(D) .
$$

Since $P_{0}(s) \neq 0$ on $R^{n}$, we take $\mathscr{F}\{u\}(s)$ to be $\mathscr{F}\left\{u_{r}\right\}(s) / P_{0}(s)$, which can be extended to an entire function of the same exponential type as $\mathscr{F}\left\{u_{r}\right\}$. Therefore $u$ is a function in $(D)$ and $P_{0}(-i D) u=u_{r}$. Thus $P(-i D) u=f$. This completes the proof of the theorem.

COROLlary 5.1. Let the polynomial $P \in(\pi)$ be irreducible and the function $f \neq 0$ belong to $(D)$. Then the equation (12) has a solution in $(D)$ if and only if $\mathscr{F}\{f\}(s)=0$ on $N(P)$.

Remark. The sufficiency part of Corollary 5.1 appears implicitly in a proof in [9].

6. A Liouville type theorem. As an application of the results in the first four sections we obtain the homogeneous case of the equation $P(-i D) u=f$ which is a result for the Liouville type theorem in partial differential equations. The nonhomogeneous case is just the application of the results in $\S 5$. In fact, W. Littman [9] proves the similar assertions as those in Theorem 6.1 under somewhat restricted conditions. But the method used there is quite different from the symmetrization introduced in $\S 1$.

THEOREM 6.1. Let the polynomial $P$ belong to $(\Pi)$ and suppose that its irreducible factors $P_{j}$ belong to $\left(\pi_{k_{j}}\right), k_{j}>0$, if $N\left(P_{j}\right) \neq 0$. Let $u$ be a solution of the equation (12) such that $u(x)=o\left(|x|^{-d}\right)$ as $x \rightarrow \infty$ with $d \geqq n-1-\min _{1 \leqq j \leqq r} k_{j} / 2$. Then $u$ belongs to $(D)$. Furthermore $u=0$ if $f=0$.

We first give its restricted form to make its proof clear and simple in the following

Lemma 6.1. Let $P$ be irreducible and belong to $\left(\pi_{k}\right), k>0$. If $u \in S^{\prime}$ is a solution of the partial differential equation (12) and $u(x)=o\left(|x|^{-d}\right), d \geqq n-1-k / 2$, when $x \rightarrow \infty$, then $u \in(D)$. If, further, $f=0$, then $u=0$.

Proof of Theorem 6.1 (assuming Lemma 6.1). Let $u_{1}=P_{1}^{\prime}(-i D) u$ with $P_{1}^{\prime}=P / P_{1}$, $v=\mathscr{F}\{u\}$ and $v_{1}=\mathscr{F}\left\{u_{1}\right\}$. (12) implies that Supp $v \subset N(P)$ which is bounded. Let 
$\chi \in C_{c}^{\infty}\left(R^{n}\right)$ be nonnegative, $\chi=1$ on a neighborhood of $N(P)$. Then, with $\Phi_{1} \equiv \mathscr{F}^{-1}\left\{P_{1}^{\prime} \chi\right\}$,

$$
u_{1}=\mathscr{F}^{-1}\left\{P_{1}^{\prime} v\right\}=\mathscr{F}^{-1}\left\{P_{1}^{\prime} \chi * \mathscr{F}^{-1}\{v\} \equiv \Phi_{1} * u .\right.
$$

Hence $u_{1}(x)=o\left(|x|^{-d}\right)$ when $x \rightarrow+\infty$. On the other hand, $u_{1}$ is a solution of the equation $P_{1}(-i D) u_{1}=f, f \in(D)$, where $P_{1}$ is irreducible. Since

$$
d \geqq n-1-\min _{1 \leqq j \leqq r} k_{j} / 2 \geqq n-1-k_{1} / 2,
$$

Lemma 6.1. yields $u_{1} \in(D)$ and $u_{1}=0$ if $f=0$.

Repeating the above arguments $r_{1}+\cdots+r_{r}-1$ times, we see that $u$ is a solution of the equation $P_{0}(-i D) u=f_{0}, f_{0} \in(D)$. If $f_{0}=0$, taking the Fourier transform of each side shows that Supp $\mathscr{F}\{u\} \subset N\left(P_{0}\right) \neq \varnothing$, which implies $u=0$. If $f_{0} \neq 0$, then $f_{0} \in(D)$ implies that $g_{0}=\mathscr{F}\left\{f_{0}\right\}$ is an entire function of exponential type. Moreover $P_{0} v=g_{0}, N\left(P_{0}\right)=\varnothing$. Then $v(s)=g_{0}(s) / P_{0}(s)$ is well defined and entirely analytic on $C^{n}$ with the same type of growth as that of $g_{0}$. Hence $u=\mathscr{F}-1\{v\}$ belongs to $(D)$.

Proof of Lemma 6.1. First we assume $f=0$. Let $v$ be the Fourier transform of $u$. Then by taking the Fourier transform of each side of (12), we see that the support of $v$ is contained in $N(P)$. Hence by the first assertion of Theorem 4.1, if $u_{0} \neq 0$, then as $x \rightarrow \infty, u_{0}(x)=O\left(|x|^{-b}\right)$ and $u_{0}(x) \neq o\left(|x|^{-b}\right), b \leqq k / 2$. On the other hand, its last assertion implies that as $x \rightarrow+\infty, u_{0}(x)=o\left(|x|^{n-1-d-k}\right)$. Here $u_{0}$ is the inverse Fourier transform of the symmetrization $v^{\sigma}$ of $v$ with respect to $N(P)$. Since $d \geqq n-1-k / 2, n-1-k-d \leqq-k / 2 \leqq-b$. Hence $u_{0}=0$.

We claim that $u(0)=u_{0}(0)$. Denote $\chi(s) e^{i x \cdot s}$ by $E(s, x)$ and the inverse Fourier transform of $\chi E(, x)$ by $w_{0}(, x)$, where $\chi$ is the function defined in Definition 1.2. Since $E(, x)$ is in $S, w_{0}(, x)$ is in $S$ for all $x \in R^{n}$. For any $\psi \in S$, with $\varphi=\mathscr{F}\{\psi\}$,

$$
\begin{aligned}
\mathscr{F}^{-1}\left\{\chi^{2} \varphi\right\}(y) & =\left(\varphi(s), \chi^{2}(s) e^{i y \cdot s}\right)=(\varphi, \chi E(, y)) \\
& =\left(\psi, w_{0}(, y)\right)=\int_{R^{n}} \psi(x) w_{0}(x, y) d x .
\end{aligned}
$$

But from the definition, $w_{0}(x, y)=\mathscr{F}\left\{\chi^{2}\right\}(x+y)$, with $\chi^{2} \in S$. It is obvious that

$$
\int_{R^{2 n}} u(x) w_{0}(x, y) \psi(y) d(x, y)
$$

is absolutely convergent. Then by Fubini's Theorem,

$$
\begin{aligned}
(u, \psi) & =\left(v, \chi^{2} \varphi\right)=\left(u, \mathscr{F}^{-1}\left\{\chi^{2} \varphi\right\}\right) \\
& =\left(u, \int_{R^{n}} \psi(x) w_{0}(x,) d x\right)=\left(\int_{R^{n}} u(y) w_{0}(, y) d y, \psi\right) .
\end{aligned}
$$

Hence

$$
u(x)=\int_{R^{n}} u(y) w_{0}(x, y) d y
$$


On the other hand, with $w(, y)=\mathscr{F}^{-1}\left\{\chi E^{\sigma}(, y)\right\}$ as the quadratic symmetrization of $P$, Theorem 3.1 implies that

$$
u_{0}(0)=\int_{R^{n}} u(y) w(0, y) d y .
$$

But by the relation $\chi=\chi^{\sigma}$, we have

$$
\begin{aligned}
w(0, y) & =\mathscr{F}^{-1}\left\{\chi E^{\sigma}(, y)\right\}(0)=\int_{U^{\delta}} e^{i 0 \cdot s} \chi(s) E^{\sigma}(s, y) d s \\
& =\int_{U^{\delta}} \chi^{\sigma}(s) E(s, y) d s=\int_{U^{\delta}} e^{i 0 \cdot s} \chi(s) E(s, y) d s=w_{0}(0, y) .
\end{aligned}
$$

Therefore

$$
u_{0}(0)=\int_{R^{n}} u(y) w(0, y) d y=u(0)
$$

Hence $u(0)=0$. But we see that, for each $y \in R^{n}, u(x+y)$ is a solution of the equation (12). Then $u(y)=0$ for each $y \in R^{n}$. That is, $u=0$.

Next assume that $f \neq 0$ in (12). Let its Fourier transform be $g$. We claim that $g$ satisfies the condition in Corollary 5.1, i.e. condition (A) in Theorem 5.1 with $r=r_{1}=1$. Suppose, on the contrary, that there is a point $a \in N(P)$ such that $g(a) \neq 0$. Since $g$ is entirely analytic on $C^{n}$, there is a neighborhood $V$ of $a$ in $R^{n}$ on which $g(s) \neq 0$. We can assume $V \subset U^{\delta}$. Let $\varphi_{0} \in C_{c}^{\infty}\left(R^{n}\right)$ and $\chi_{j} \in C_{c}^{\infty}\left(R^{1}\right)$ be nonnegative, $\varphi_{0}(a)=1$, Supp $\varphi_{0} \subset V$ and Supp $\chi_{j} \subset[-1 / j, 1 / j]$. Set $\mathrm{Cl}(\chi(s))$ $=g(s) \varphi_{0}(s)$. Then $\chi \in C_{c}^{\infty}\left(R^{n}\right), \chi g \geqq 0$, and $\chi g>0$ on some neighborhood $V_{1}$ of $a$ such that $\bar{V}_{1} \subset V$. By (12) $P v=g$. We have for all $j>0, P\left[v \chi_{\chi_{j}}(P)\right]=g \chi \chi_{j}(P)$, which is positive at $a$. Making the symmetrization with respect to $P$ on both sides, we get

$$
q[v \chi]^{o}(q) \chi_{j}(q)=[g \chi]^{o}(q) \chi_{j}(q) .
$$

Let $j \rightarrow \infty$; since $\chi_{j}(q)$ converges to the Dirac-measure $\delta(q)$, we have $0=[g \chi]^{\rho}(0)>0$. This is impossible. Hence $g$ satisfies the condition in Corollary 5.1. Therefore there is $u_{1} \in(D)$ which is a solution of (12). But $u-u_{1}$ satisfies the condition for the case $f=0$. We then have $u=u_{1} \in(D)$. This is the assertion of the lemma.

\section{BIBLIOGRAPHY}

1. A. S. Besicovitch, Almost periodic functions, Cambridge Univ. Press, Cambridge, 1932.

2. A. Friedman, Generalized functions and partial differential equations, Prentice-Hall, Englewood Cliffs, N. J., 1963. MR 29 \#2672.

3. - Entire solutions of partial differential equations with constant coefficients, Duke Math. J. 31 (1964), 235-240. MR 28 \#5255.

4. I. M. Gel'fand and G. E. Silov, Generalized functions. Vol. 1: Properties and operations, Fizmatgiz, Moscow, 1958; English transl., Academic Press, New York, 1964. MR 20 \#4182; MR 29 \#3869.

5. - Generalized functions. Vol. 2: Spaces of fundamental and generalized functions, Fizmatgiz, Moscow, 1958; English transl., Academic Press, New York, 1968. MR 21 \#5142a; MR 37 \#5693. 
6. I. M. Gel'fand and G. E. Silov, Generalized functions. Vol. 3: Theory of differential equations, Fizmatgiz, Moscow, 1958; English transl., Academic Press, New York, 1967. MR 21 \#5142b; MR 36 \#506.

7. I. M. Gel'fand and Z. Ja. Sapiro, Homogeneous functions and their extensions, Uspehi Mat. Nauk 10 (1955), no. 3 (65), 3-70; English transl., Amer. Math. Soc. Transl. (2) 8 (1958), 21-85. MR 17, 371; MR 20 \#1061.

8. W. Littman, Fourier transforms of surface-carried measures and differentiability of surface averages, Bull. Amer. Math. Soc. 69 (1963), 766-770. MR 27 \#5086.

9. - Decay at infinity of solutions to partial differential equations with constant coefficients, Trans. Amer. Math. Soc. 123 (1966), 449-459. MR 33 \#6110.

10. C. Roumieu, Ultra-distributions définies sur $R^{n}$ et sur certaines classes de variétés différentiables, J. Analyse Math. 10 (1962/63), 153-192. MR 28 \#1487.

11. L. Schwartz, Théorie des distributions. Tomes 1, 2, Actualités Sci. Indust., no. 1091, 1122, Hermann, Paris, 1950, 1951. MR 12, 31; 833.

12. J. F. Tréves, Lectures on linear partial differential equations with constant coefficients, Notas de Matemática, no. 27, Inst. Mat. Pura e Apl. do Conselho Nacional de Pesquisas, Rio de Janeiro, 1961. MR 27 \#5020.

13. - Linear partial differential equations with constant coefficients. Existence, approximations and regularity of solutions, Math. and its Applications, vol. 6, Gordon and Breach, New York, 1966. MR 37 \#557.

14. —_ Differential polynomials and decay at infinity, Bull. Amer. Math. Soc. 66 (1960), 184-186. MR 22 \#8227.

15. R. E. Williamson, R. H. Crowell and W. Trotter, Calculus of vector functions, 2nd ed., Prentice-Hall, Englewood Cliffs, N. J., 1968.

NoRTHWESTERN University, Evanston, IllinoIs 60201

Louisiana State University, New Orleans, Louisiana 70122 